\title{
Therapeutic Shifts in the Management of Cancer-Associated Thrombosis
}

\author{
Walter Ageno ${ }^{1}$ \\ ${ }^{1}$ Department of Medicine and Surgery, University of Insubria, \\ Varese, Italy
}

Thromb Haemost 2017;117:2012.

Venous thromboembolism (VTE) is the second leading cause of death in cancer patients and affects 5 to $20 \%$ of patients with known cancer. ${ }^{1}$ The treatment of cancer-associated VTE is challenging because the risk of recurrence and bleeding is higher in these patients than in other patient groups. ${ }^{2}$ One of the explanations for such higher rates was the problematic management of anticoagulant therapy with the vitamin $\mathrm{K}$ antagonists (VKA). Drug interactions, liver dysfunction and malnutrition often lead to unpredictable responses to VKA, while the need for invasive procedures and the occurrence of side effects of chemotherapeutic agents require frequent treatment interruptions that are difficult to manage with VKA given their slow onset and offset of action. To overcome these limitations, low-molecular-weight heparin (LMWH) was proposed as a therapeutic alternative in this setting and several studies have consistently proven its superior efficacy over VKA with similar rates of major bleeding. ${ }^{3}$ Following the recommendations of several international guidelines, LMWH has become the standard of practice for cancer-associated VTE in many countries. To assess the impact of this therapeutic shift, den Exter and colleagues have performed a retrospective, observational study comparing major clinical outcomes in patients with cancer-associated VTE before and after the recommendation of LMWH as the treatment of choice in this setting. ${ }^{4}$ In this study, while the use of LMWH raised from 23 to $67 \%$, the rates of recurrent VTE or major bleeding remained unchanged, suggesting that multiple clinical factors
Address for correspondence Walter Ageno, Department of Medicine and Surgery, University of Insubria, Varese, Italy (e-mail: walter.ageno@uninsubria.it).

rather than the anticoagulant agent per se contribute to the occurrence of these clinical outcomes. This report provides important information from an unselected population. However, these findings should not be overestimated given the design of the study, which cannot challenge the results of randomized controlled trials, and because major changes in the treatment of cancer may have affected the baseline risk of VTE and bleeding in these patients overtime. Some practical advantages with the use of LMWH remain undisputable, but are now challenged by the direct oral anticoagulants.

\section{References}

1 Chew HK, Wun T, Harvey D, Zhou H, White RH. Incidence of venous thromboembolism and its effect on survival among patients with common cancers. Arch Intern Med 2006;166(04):458-464

2 Prandoni $\mathrm{P}$, Lensing AW, Piccioli $\mathrm{A}$, et al. Recurrent venous thromboembolism and bleeding complications during anticoagulant treatment in patients with cancer and venous thrombosis. Blood 2002;100(10):3484-3488

3 Akl EA, Kahale L, Barba M, et al. Anticoagulation for the long-term treatment of venous thromboembolism in patients with cancer. Cochrane Database Syst Rev 2014;(07):CD006650

4 den Exter P, Hooijer J, van der Hulle T, van Oosten J, Dekkers O, Klok E, Huisman M. Vitamin K antagonists compared to low molecular weight heparins for treatment of cancer associated venous thromboembolism: an observational study in routine clinical practice. Thromb Haemost 2017;117(11):2163-2167
T\&H Insights on den Exter et al. Thromb Haemost 2017;117: 2163-2167. received

September 15, 2017

accepted

September 15, 2017
DOI https://doi.org/ 10.1160/TH17-09-0635. ISSN 0340-6245. 